\title{
Energy Spectrum of Shallow Donor in Elongated InAs/GaAs Volcano-Shaped Quantum Dot
}

\author{
L. F. García ${ }^{1}{ }^{1}{ }^{1}$ R. A. Escorcia, ${ }^{2}$ and I. D. Mikhailov ${ }^{1}$ \\ ${ }^{1}$ Escuela de Física, Universidad Industrial de Santander, A. A. 678, Bucaramanga, Colombia \\ ${ }^{2}$ Grupo de Investigación en Teoría de la Materia Condensada, Universidad de Magdalena, Santa Marta, Colombia
}

Correspondence should be addressed to L. F. García; 1fragar@gmail.com

Received 24 January 2018; Accepted 26 February 2018; Published 4 April 2018

Academic Editor: Yuri Galperin

Copyright ( 92018 L. F. García et al. This is an open access article distributed under the Creative Commons Attribution License, which permits unrestricted use, distribution, and reproduction in any medium, provided the original work is properly cited.

\begin{abstract}
We study the effect of the nonparabolicity of the conduction band on the spectral properties of the shallow donor in elongated InAs/GaAs quantum dots with volcano-shaped profile in the framework of the Kane model by using a simple semiempiric relation between geometrical parameters of the quantum dot profile and the confinement potential, governing the in-plane electron's movement. We represent the solution of the Schrödinger equation in the form of double Bessel-Fourier series expansion. We show that the nonparabolicity of dispersion of the conduction band, given by the Kane formula, conduces to significant lowering of the donor energies and to stronger confinement of the electron within the quantum dot. Calculated results for the energies as functions of the electric and magnetic fields for different quantum dot dimensions were compared with those obtained in the effective mass approximation. Our results exhibit a high sensibility of the probability density of electron distribution in volcano-shaped quantum dot to the variation of the external magnetic and electric fields.
\end{abstract}

\section{Introduction}

Recent development of nanofabrication techniques such as chemical vapor deposition, liquid-phase, molecular beam, and advanced droplet epitaxy [1-6] offers an attractive route for designing of new semiconductor materials for possible applications in opto- and microelectronic devices. In addition, the electrical and optical properties of nanostructures may be modified by doping the shallow impurities, whose energy levels in these materials are very sensible to the variation of the impurity position, the nanostructure size, and dimension. A great deal of attention of investigators during previous two decades has been attracted to the study of the effect of the quantum confinement on the impurity states in various nanostructures, such as single, double, and multiple quantum wells [7-11], quantum-well wires $[12,13]$, and quantum dots (QDs) [14-18] by commonly using the effective mass approximation (EMA) and the variational method.

The morphology of nanostructures can be sufficiently complicated, and therefore the calculation by means of the variational method requires generally a lot of computational work related especially to the estimation of multidimensional integrals. In the case of QDs, which generally have a form of nonhomogeneous very thin layer, the numerical procedure can be simplified in the framework of the adiabatic approximation (AA), in which one can study separately the slow inplane and fast transversal movements [19,20]. Application of the AA allows us to reduce the actual 3D problem for impurity confined in a thin semiconductor layer to two-dimensional Schrödinger equation for the hydrogen atom placed in a field with an adiabatic potential. The adiabatic potential of QDs with different morphologies can be calculated numerically [21].

However, the applicability of two-dimensional models and the AA in many cases is doubtful for two reasons: possible nonparabolicity of the conduction band and strong tunneling of the electron outside the QD trough junctions. The first of them is essential when the gap $\Delta$ between the valence and conduction bands is small and the mixing of these two bands is essential such as in $\operatorname{InAs}(\Delta \sim 0.4 \mathrm{eV})$ or $\operatorname{InSb}(\Delta \sim 0.5 \mathrm{eV})$, while the tunneling becomes essential when the barrier 
height in junctions and the layer thickness are relatively small. Calculations, realized previously in framework of the EMA [22] have demonstrated that in a thin InAs/GaAs quantum disk with the thickness $<4 \mathrm{~nm}$, the electron can be located outside the disk with the probability being about $30 \%$. Earlier, it has been proposed to replace the EMA by the Kane model [23] with a consistent energy-dependent effective mass in order to analyze these effects on the spectral properties of the electron and exciton confined in a thin InAs/GaAs QD by using finite element method $[24,25]$ and AA [26]. It has been shown that the nonparabolicity causes an essential lowering of the carriers' energies reinforcing in this way their confinement inside SAQD and giving in this way an additional reason for applicability of 2D models for QDs with relatively small barrier heights and energy gaps.

Recently, advanced technics have enabled the fabrication quantum dot structures with a wide diversity of almost ideal morphologies but with inherent imperfections, reflected in experimentally found photoluminescence spectra [27-31]. The structures generally show a good circular symmetry, but with an inherent slight elongation along of one of the directions and a noticeable asymmetry in the thickness of the layer breaking the rotational symmetry, which complicate the theoretical analysis of the photoluminescence spectrum. Below we propose a simple numerical procedure, which allows us to analyze the energy spectrum of a shallow donor placed in an elongated InAs/GaAs volcano-shaped quantum dot, in the framework of the Kane model and by using a nonvariational method. Moreover, our procedure permits studying the effect of the electric and magnetic fields applied, respectively, perpendicularly and parallel to the crystal growth direction. In order to calculate the energies of lower levels as functions of the magnetic field, we use an interpolative relationship between the adiabatic potential of the InAs layer and the local thickness found in [26], considering a model with finite barrier height in the junctions of the InAs QD surrounded by the GaAs matrix and taking into account the nonparabolicity of the conduction bands in both materials. We obtain the energies and wave functions of reduced two-dimensional Schrödinger equation by using the double Fourier-Bessel series expansion method.

The organization of this paper is as follows: In Section 2, we present the theoretical model and explain our calculation method of the energies, the charge distribution, the density of energy states, and the electric and magnetic momenta for on- and off-axis donors confined in an elongated InAs/GaAs volcano-shaped quantum dot. Numerical results are presented in Section 3 and some conclusions in Section 4.

\section{Theory}

In order to study the energy spectrum of shallow donor confined in volcano-shaped InAs/GaAs QR, taking into account strong nonparabolicity of the InAs conduction band, we use below a simple version of the Kane model, proposed in $[23,24]$. In this version the electron in a semiconductor bulk is considered as a free particle with the energy-dependent effective mass placed in a band with the parabolic dispersion. In addition, it is assumed that the material parameters in
QDs have mismatches in junctions and they are characterized by spatial-dependent band-gap energy and effective mass. The corresponding nonlinear Schrödinger equation for the electron released from the donor with the position vector $\mathbf{r}_{i}$ in a homogeneous magnetic field $\mathbf{B}=B \widehat{\mathbf{z}}$ applied along the $z$-axis and a homogeneous electric field $\mathbf{F}=F \widehat{\mathbf{x}}$ in the Kane model has the form $[23,24]$

$$
\begin{aligned}
& \widehat{H}(E, \mathbf{r}) \Psi(\mathbf{r})=E \Psi(\mathbf{r}) ; \\
& \widehat{H}(E, \mathbf{r}) \\
& =(-i \hbar \nabla+e \mathbf{A}(\mathbf{r})) \frac{1}{2 m *(E, \mathbf{r})}(-i \hbar \nabla+e \mathbf{A}(\mathbf{r})) \\
& \quad+V(\mathbf{r})+e F x-\frac{e^{2}}{4 \pi \varepsilon \varepsilon_{0}\left|\mathbf{r}-\mathbf{r}_{i}\right|} .
\end{aligned}
$$

Here $\varepsilon$ is the dielectric constant of the InAs, $\mathbf{A}=\mathbf{B} \times \mathbf{r} / 2$ is vector potential, $V(\mathbf{r})$ is the confinement potential equal to zero inside the QD layer $0<z<d(\rho, \varphi)$ and to $V_{0}$; otherwise, $d(\rho, \varphi)$ defines the profile of $\mathrm{QD}$, and $m *(E, \mathbf{r})$ is the energyand position-dependent effective mass, defined as

$$
\frac{m_{0}}{m *(E, \mathbf{r})}=\frac{2 P^{2}}{3 \hbar^{2}}\left(\frac{2}{E_{g}(\mathbf{r})+E}+\frac{1}{E_{g}(\mathbf{r})+E+\Delta(\mathbf{r})}\right),
$$

where $m_{0}$ is free electron mass, $P$ is Kane's momentum matrix element, $E_{g}$ is the band gap, $\Delta$ is the spin-orbit splitting of the valence band, and $E$ are eigenvalues of the nonlinear wave equation (1). We simulate the InAs/GaAs ring by using the material parameters for InAs inside the rings being $E_{g}^{(1)}$ $=0.42 \mathrm{eV}, \Delta_{1}=0.38 \mathrm{eV}$, and $m_{1} *(0)=0.024 m_{0}$. The parameters for GaAs are $E_{g}^{(2)}=1.52 \mathrm{eV}, \Delta_{2}=0.34 \mathrm{eV}$, and $m_{2} *(0)=0.067 m_{0}$; the mismatch of the band gap at the junction is equal to $V_{0}=0.7\left(E_{g}^{(2)}-E_{g}^{(1)}\right)$ [27-31]. We use below, as dimensionless units, the effective Bohr radius $a_{0}^{*}=4 \pi \varepsilon_{0} \varepsilon \hbar^{2} / m_{1}{ }^{*}(0) e^{2} \approx 28.65 \mathrm{~nm}$, the effective Rydberg $R_{y}^{*}=e^{2} / 8 \pi \varepsilon_{0} \varepsilon a_{0}^{*} \approx 1.93 \cdot 10^{-3} \mathrm{eV}$, and $\gamma=e \hbar B /$ $2 m_{1}{ }^{*}(0) R_{y}^{*} \approx 1.26 \cdot B(T) \alpha=e a_{0} * F / R y * \approx 1.48 F(\mathrm{kV} /$ $\mathrm{cm})$ as units of magnetic and electric fields and the dimensionless magnetic field strength, respectively, with $\varepsilon$ being the dielectric constant of InAs material.

Taking into account the strong anisotropy of the nanostructure, one can use the adiabatic approximation, in which fast transverse and slow in-plane movements may be considered separately, representing the electron wave function as follows:

$$
\begin{aligned}
\Psi(\mathbf{r}) & =\left.f_{\rho, \varphi}(z) \psi(\rho, \varphi) \cup \widehat{H}\right|_{\rho, \varphi}\left(E_{z}, \mathbf{r}\right) f_{\rho, \varphi}(z) \\
& =E_{z}(\rho, \varphi) f_{\rho, \varphi}(z) .
\end{aligned}
$$

The transverse wave function $f_{\rho, \varphi}(z)$ with fixed polar coordinates $\rho, \varphi$ satisfies additional Ben Daniel Duke boundary condition at the bottom of the layer with the coordinate $z=0$ and at each point of the top of the layer $z=d(\rho, \varphi)$. One can find the lowest energy $E_{z}(\rho, \varphi)$ for such movement that is used then as the adiabatic potential for the in-plane electron 


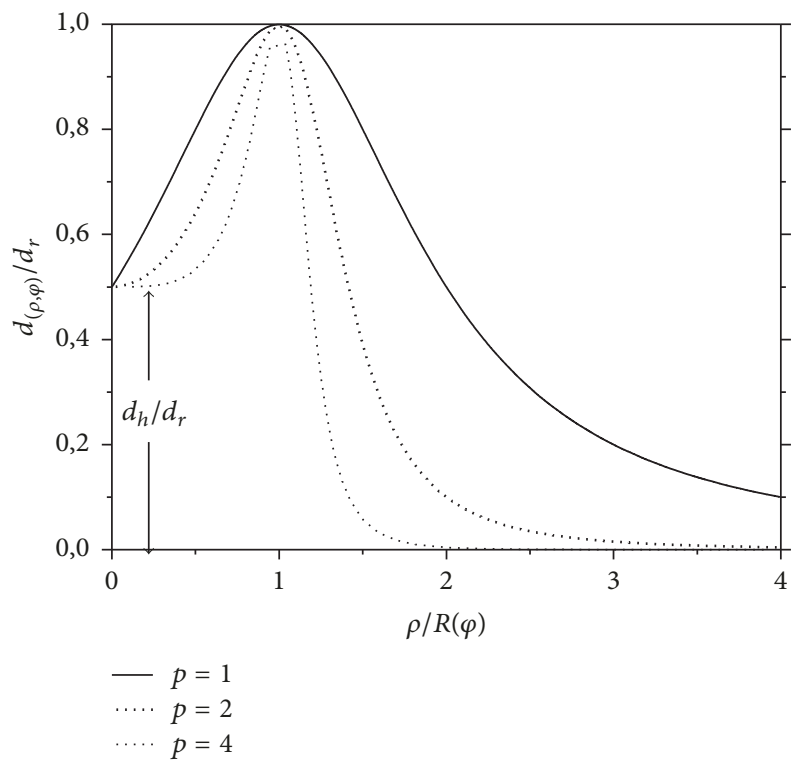

FIgURE 1: Profiles of radial cross sections.

movement $V(\rho, \varphi)$ by solving a transcendental equation [26] and obtaining the in-plane adiabatic confinement potential related to the layer thickness $d(\rho, \varphi)$ as follows:

$$
V(\rho, \varphi)=\frac{9.5}{d(\rho, \varphi)} .
$$

To simulate a uniform volcano-like $\mathrm{QR}$ we use below the parameterized function, which in polar coordinates defines the variation of the thickness of the layer:

$$
\begin{array}{r}
d(\rho, \varphi)=d_{r}\left\{1+\left[\frac{2\left((\rho / R(\varphi))^{p}-1\right)}{w}\right]^{2}\right\}^{-1} ; \\
p=1,2,3, \ldots
\end{array}
$$

Here $d_{r}$ is the height of the rim and $w$ is the effective width of the volcano, related to the central hole $d_{h}$ as follows:

$$
d_{h}=\frac{d_{r}}{\left[1+(2 / w)^{2 p}\right]} ; \quad w=2 \cdot\left(\frac{d_{r}}{d_{h}-1}\right)^{-1 / 2 p} .
$$

We choose the dependence $R_{r}(\varphi)$ of the oval-shaped rim radius with the eccentricity $e$ on the polar coordinate $\varphi$ as

$$
R_{r}(\varphi)=\frac{a}{\left(1+e \cos ^{2} \varphi\right)} ; \quad e \geq 0
$$

In Figure 1 we display profiles of radial cross sections of the model for different central hole heights, and Figure 2 shows the $3 \mathrm{D}$ images corresponding to the ratio $d_{h} / d_{r}=0.1$ at the upper row, the ratio $d_{h} / d_{r}=0.4$ at the lower row, and the eccentricities $e=0,0.5$, and 1.0 .

The differential equation for the in-plane part of the wave function (3) $\psi(\rho, \varphi)$ in the two-dimensional field resulted from the adiabatic potential (4) and the Coulomb attraction to the donor located over the $X$-axis, with the position vector in dimensionless units having the following form [27]:

$$
\begin{aligned}
& H_{\|}(\rho, \varphi) \psi(\rho, \varphi)=\widetilde{E} \psi(\rho, \varphi) ; \\
& \psi(\rho, \varphi+2 \pi)=\psi(\rho, \varphi) ; \\
& \widetilde{E}=E \cdot \eta(E) ; \\
& \eta(E)=\left(\frac{E}{E_{g}^{(1)}}+1\right)\left[\frac{E}{\left(E_{g}^{(1)}+\Delta_{1}\right)}+1\right] \\
& \cdot\left[\frac{E}{\left(E_{g}^{(1)}+2 \Delta_{1} / 3\right)}+1\right]^{-1}, \\
& H_{\|}(\rho, \varphi)=\widehat{T}(\rho, \varphi)-i \gamma \frac{\partial}{\partial \varphi}+\frac{\gamma^{2} \rho^{2}}{4}+\alpha F \rho \cos \varphi \\
& \quad+\widehat{U}(\rho, \varphi) ; \\
& \widehat{T}(\rho, \varphi)=-\frac{\partial^{2}}{\partial \rho^{2}}-\frac{1}{\rho} \frac{\partial}{\partial \rho}-\frac{1}{\rho^{2}} \frac{\partial^{2}}{\partial \varphi^{2}} ; \\
& \widehat{U}(\rho, \varphi)=\frac{9.5}{d(\rho, \varphi)}-\frac{2}{\sqrt{\rho^{2}+\rho_{i}^{2}-2 \rho_{i} \rho \cos \varphi}} .
\end{aligned}
$$

The boundary value problem for the wave equation (8a) and ( $8 \mathrm{~b}$ ) should be completed by frontier condition at the radial direction. In our calculations, we assume that the wave function is equal to zero outside the circular region of the radius $R_{F}$; that is, $\psi\left(R_{F}, \varphi\right)=0$.

To solve eigenvalue problem ( $8 \mathrm{a})$ and ( $8 \mathrm{~b})$ by means of the double Fourier-Bessel series expansion method, one can use the following normalized base functions that automatically fulfill the frontier conditions:

$$
\begin{aligned}
\psi_{m, n}(\rho, \varphi)=\frac{A_{m, n}}{R \sqrt{\pi}} e^{i m \varphi} J_{m}\left(\frac{x_{n, m} \rho}{R}\right): & \\
A_{m, n} & =\frac{1}{J_{m+1}\left(x_{m, n}\right)} ;
\end{aligned}
$$

$$
\left\langle\psi_{m, n} \psi_{m^{\prime}, n^{\prime}}\right\rangle=\delta_{m, m^{\prime}} \delta_{n, n^{\prime}}
$$

Here $J_{m}(x)$ is the Bessel function of the order $m$ and $x_{n, m}$ is the $n$th zero of this function. We seek the solution of (8a) and (8b) in the form of the following series expansion:

$$
\psi(\rho, \varphi)=\sum_{n, m=-\infty}^{\infty} C_{n, m} \psi_{m, n}(\rho, \varphi) .
$$

According to the Galerkin projection method, functions (9a) and (9b) satisfy conditions 


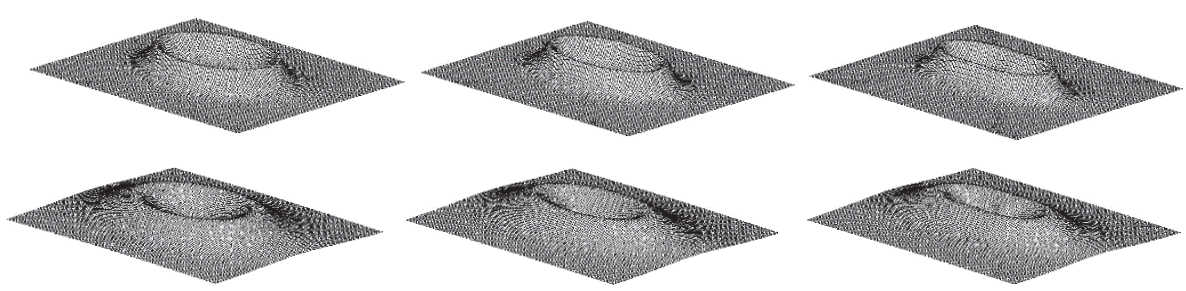

FIGURE 2: 3D images of elongated volcano-shaped QDs with different eccentricities; $e=0,0.5$, and 1.0.

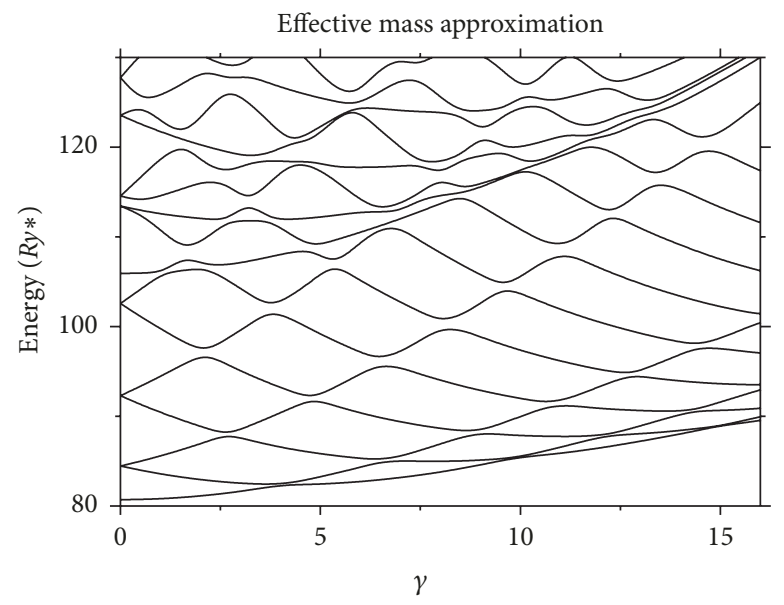

(a)

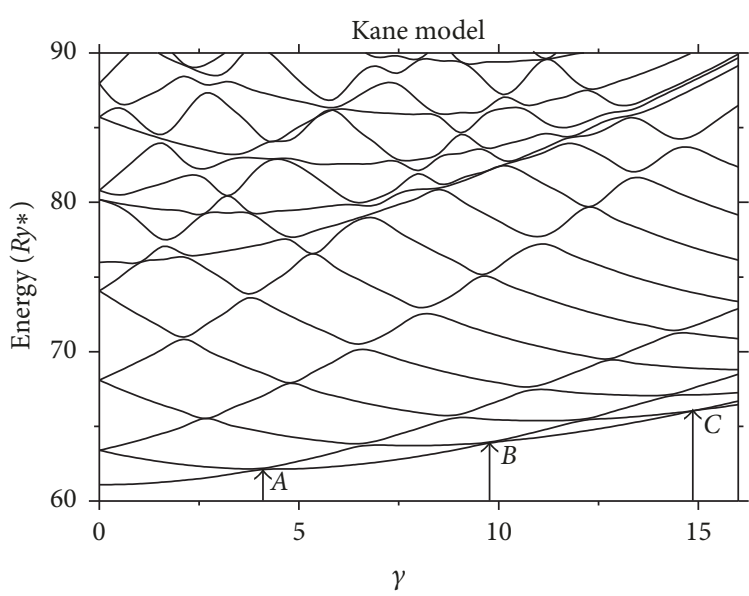

(b)

FIGURE 3: Energies of the on-center donor in circular QR as functions of the external magnetic field calculated in (a) in the effective mass approximation and (b) in the framework of the Kane model.

$$
\begin{aligned}
\left\langle\psi_{m, n}(\rho, \varphi)|\widehat{H}(\rho, \varphi)-\widetilde{E}| \psi(\rho, \varphi)\right\rangle= & 0 ; \\
& \widetilde{E}=E \cdot \eta(E) .
\end{aligned}
$$

Substituting (9a) and (9b) in (10), one can obtain the following system of linear secular equations:

$$
\begin{gathered}
\sum_{n, m=-\infty}^{\infty}\left(H_{n, m ; n^{\prime}, m^{\prime}}-\widetilde{E} \delta_{m, m^{\prime}} \delta_{n, n^{\prime}}\right) C_{n^{\prime}, m^{\prime}}=0, \\
H_{n, m ; n^{\prime}, m^{\prime}}=\frac{A_{m, n} A_{m, n^{\prime}}}{\pi R^{2}} \int_{0}^{2 \pi} d \varphi \int_{0}^{R} e^{-i m \varphi} J_{m}\left(\frac{x_{m, n} \rho}{R}\right) \\
\cdot \widehat{H}_{\|}(\rho, \varphi) e^{-i m^{\prime} \varphi} J_{m^{\prime}}\left(\frac{x_{m^{\prime}, n^{\prime}} \rho}{R}\right) \rho d \rho .
\end{gathered}
$$

We analytically found matrix elements (11b) for all terms of the Hamiltonian (8b), except the last one, which was calculated numerically. By solving the secular equation (11a), we found eigenvalues $\widetilde{E}_{k}$ and eigenvectors $C_{n m}^{(k)}, k=1,2,3, \ldots$ Eigenvalues $\widetilde{E}_{k}$ define the donor energies in the effective mass approximation. In order to take into consideration the effect of the nonparabolicity of the conduction band and to find the corresponding energies $E_{k}$ in the framework of the Kane model, one should solve, in addition, nonlinear algebraic equations $E_{k} \cdot \eta\left(E_{k}\right)=\widetilde{E}_{k}$.

Once the secular equation is solved, eigenvalues $\widetilde{E}_{k}$ and eigenvectors $C_{n m}^{(k)}, k=1,2,3, \ldots$, are found, and then the probability density of charge distribution of the corresponding states can be found as follows:

$$
\begin{aligned}
& f^{(k)}(\rho, \varphi)=\left(\pi R^{2}\right)^{-1} \\
& \cdot \sum_{n, m, n^{\prime}, m^{\prime}} C_{n m}^{(k)^{*}} C_{n^{\prime} m^{\prime}}^{(k)} A_{m, n} A_{m^{\prime}, n^{\prime}} J_{m}(t) J_{m^{\prime}}(t) \\
& \cdot \cos \left(m-m^{\prime}\right) \varphi ; \quad t=\frac{x_{n, m} \rho}{R} .
\end{aligned}
$$

\section{Results and Discussions}

All results that we present below are referred to as the structure with geometrical parameters: $a=20 \mathrm{~nm}, d_{r}=4 \mathrm{~nm}$, $d_{h}=2 \mathrm{~nm}$, and $p=1$. First, we analyze the effect of the nonparabolicity of the conduction band.

In Figure 3 we compare the curves of the energies dependencies on the external magnetic field calculated for on-center donor in the circular QR $(e=0)$ in the effective mass approximation and in the framework of the Kane model. Similitude of the results obtained in both models is seen with only one essential difference: the energies in the Kane model are essentially lower than those in the effective mass approximation; that is, the nonparabolicity of the conduction band softens the effect of the structural confinement and in this way offers an additional argument for the applicability of the adiabatic approximation [26]. 


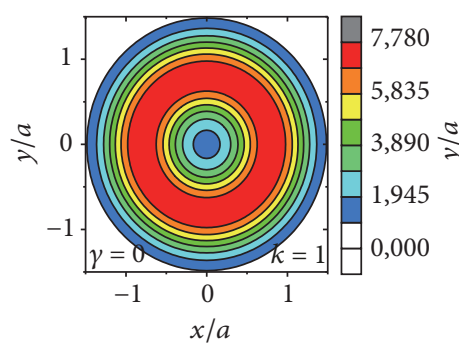

(a)

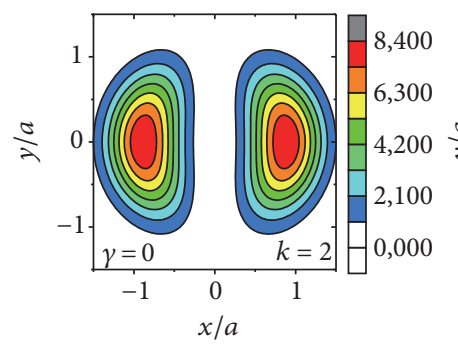

(b)

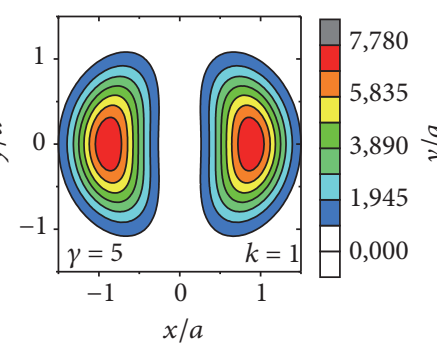

(c)

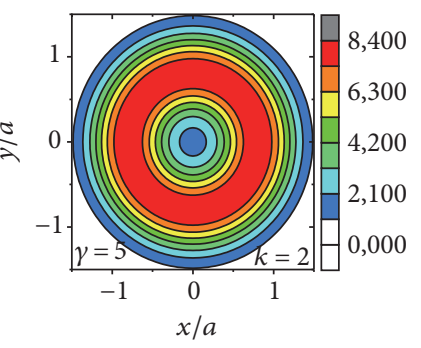

(d)

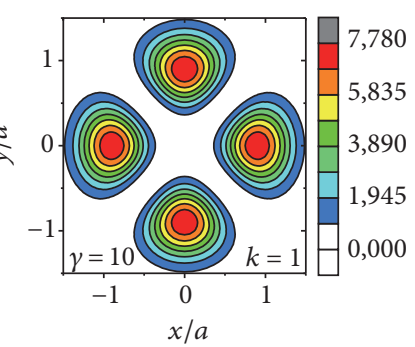

(e)

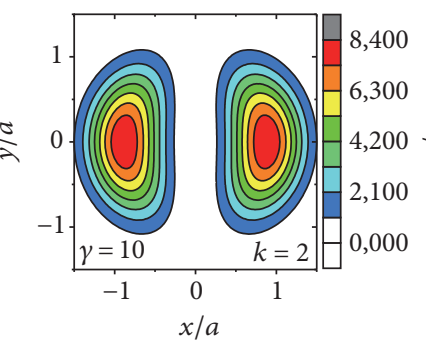

(f)

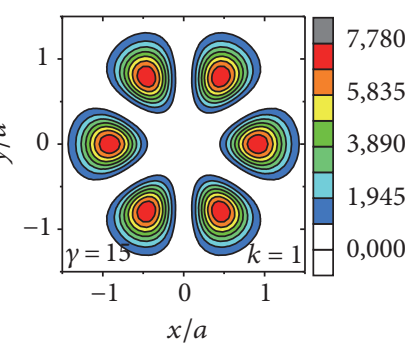

(g)

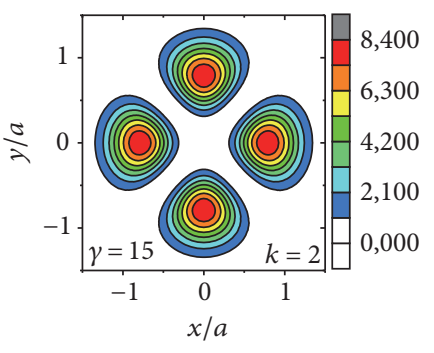

(h)

FIGURE 4: Charge distribution for on-center donor in the ground state (a, c, e, g) and in the first excited state (b, d, f, h) for $\gamma=0,5,10,15$ in circular QR.

In Figures 3(a) and 3(b) one can see that the dependence of the ground state energy on the magnetic field is feebly manifested only in a weak oscillation of the ground state energy near a straight line. In contrast, the spatial charge distribution and the magnetic momentum can suffer sharp variations because the energy gaps between the ground and first excited states periodically vanish. At points $A, B$, $C$, where gaps in Figure 3(b) are vanished, the values of the magnetic fields approximately are $\gamma=5,10$, and 15, respectively. Therefore, we calculate for these values the probability density of charge distribution in the ground $(k=$ $1)$ and first excited $(k=2)$ states by using the relation (12). Corresponding contour plots are displayed in Figure 4, for the ground state in Figures 4(a), 4(c), 4(e), and 4(g) and for the first excited state at Figures 4(b), 4(d), 4(f), and 4(h). The charge distributions in all cases are similar to those of two-dimensional orbits hydrogen-like. For example, for zeromagnetic field case the charge distribution in the ground state is $s$-like and for excited state in Figure $4(\mathrm{~b})$ it is $p$-like. As the magnetic field is increased up to $\gamma=5$ at the point $A$ in Figure 3, the charge distributions in the ground and excited states in Figures 4(c) and 4(d) are inverted. Similar transformations suffer charge distributions as the increasing magnetic field reaches the point $B$ and the value $\gamma=10$; the $p$-like charge distribution of the ground state is transformed in the $d$-like one, while the charge $s$-like distribution of the excited state is transformed in in $p$-like one in Figures 4(e) and $4(\mathrm{f})$, respectively.

3.1. Off-Center Donor in Circular QR. The displacement of the donor from the center of the circular QR breaks the system axial symmetry of the structure and provides the localization of the lowest rotational state, manifested in removing of the periodic degeneration of two lowest levels and in a substantial increase of the nonvanishing gap between them as the distance from the position of the donor to the center is increased. In Figure 5 we show lower energies as functions of the magnetic field for off-center donors placed at the distances $\rho_{i}=10 \mathrm{~nm}$ and $\rho_{i}=20 \mathrm{~nm}$ from the center of the circular QR.

It is seen that the energy dependencies of the upper excited states on the magnetic field are changed with the displacement of the donor position insignificantly. Commonly, the break of the symmetry of the structures disturbs the charge distribution to a much greater extent than energy spectrum. In Figure 6 we display contour plots that show the evolution of the charge distribution in QR with off-center donor in the ground state under growing magnetic field.

It is seen that the increase of the magnetic field does not change the symmetry of the charge distribution in the ground state, providing only the spatial confinement around the donor location without mixing of the ground state with excited states.

3.2. On-Center Donor in Elongated $Q R$. Donor energies calculated as functions of the magnetic field in elongated QRs and presented in Figure 7 exhibit dependencies essentially different from the structure with circular rim shown in Figure 3. In elongated $Q R$, the central symmetry $C_{\infty}$ of the confinement potential is transformed to the axial symmetry $D_{2}$, for which only the projection of the angular momentum on the horizontal axis is conserved. It allows us to classify the donor states in elongated QRs according to the value $\Lambda=$ $0,1,2$ of this projection, commonly denoted by letters $\Sigma, \Pi, \Delta$, respectively. Moreover, this structure is invariant with respect to the inversion operation; that is, the Hamiltonian remains unchanged if signs of all electron coordinates are turned over. Since this transformation commutes also with the operator 


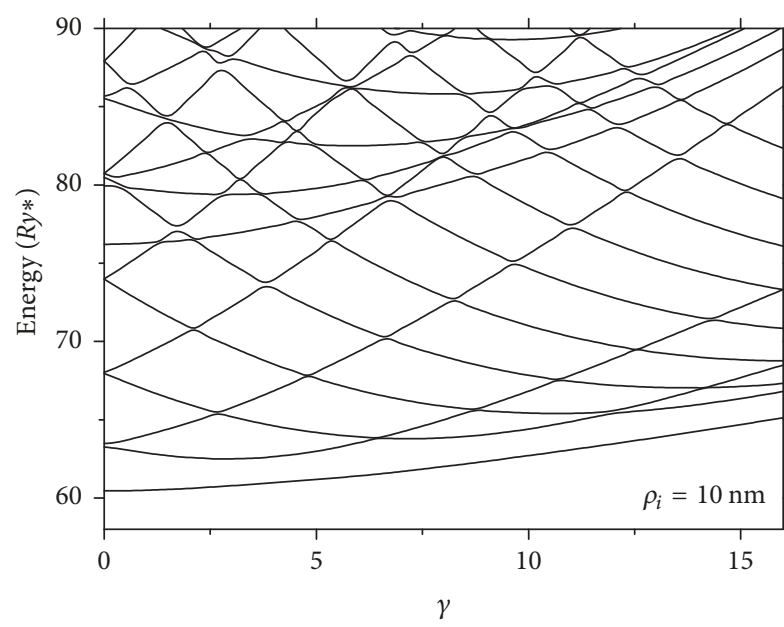

(a)

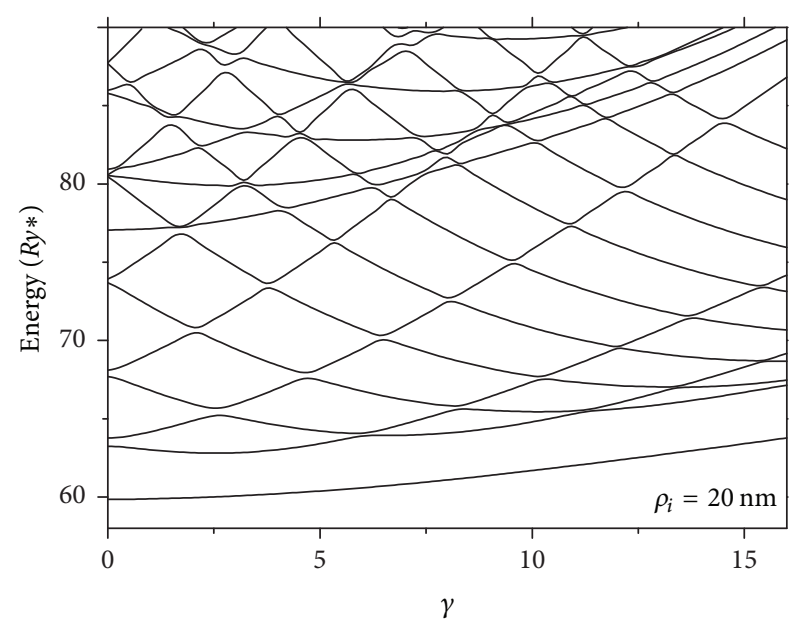

(b)

FIGURE 5: Energies of the off-center donor in circular QR as functions of the external magnetic field calculated for two different donor's positions.

of the angular momentum, there are two different wave functions corresponding to the same angular momentum $\Lambda$ but to two different wave functions, one with even $(g)$ parity, which is unchanged with respect to the inversion, and the other with odd $(u)$ parity, which changes sign.

Unlike the corresponding dependencies for circular QR in Figure 3, one can observe in Figure 7 for $\gamma=0$ nonzero gaps between the states with different parity $\Sigma_{g}, \Sigma_{u}$ and $\Pi_{g}$, $\Pi_{u}$, in the energy spectrum of on-center donor. The splitting of sublevels with different parities in this case is induced by the anisotropy of the confinement potential in elongated QR. This degeneracy is due to the twofold rotational symmetry of the structure, but unlike the similar effect in circular QR, the degeneracy is no longer restored periodically. However, these two sublevels become again degenerated when the magnetic field is increased. The greater the eccentricity parameter $e$ is, that is, the smaller the lateral dimension of the $\mathrm{QR}$ is, the larger then the distances between adjacent points of the degeneration in the lower curves in Figure 8 become. (Note that in circular $\mathrm{QR}$ of radius $R$ these distances are $\sim 1 / R^{2}$.)

One can reveal another interesting peculiarity of the curves in Figure 7 compared with similar dependencies in Figure 5 for off-axis donor in the circular QR, a system with similar symmetry. The presence of the off-axis donor in circular $\mathrm{QR}$ also breaks the central symmetry $C_{\infty}$ of the structure, resulting in the localization of the electron in the ground state and the formation of a nonvanishing energy gap between two lowest levels. One can observe a similar alteration of the energy spectrum at the lower part of the energies dependencies in Figure 7 where, unlike Figure 5, not one but two curves for states $\Sigma_{g}$ and $\Sigma_{u}$ are split off from other upper energy levels. The splitting of these two levels is due to the additional symmetry that is inherent in the geometry of the elongated QR with on-center donor.

The existence of the intersections of the energies dependencies of the ground and the excited states on the magnetic field conduces to a drastic alteration of the charge distribution in the system, when the magnetic field exceeds a critical value. As an example, let us consider two curves presented in Figure $7(\mathrm{~b})$ for states $\Sigma_{g}$ and $\Sigma_{u}$, which have the first intersection at the point $A$ as the magnetic field increases reaching the value $\gamma=10$. At this point configurations of the ground and first excited states are changed sharply. In order to compare these configurations before and after this transition we present in Figure 8 the contour plots of the charge distributions in two lower states for the external magnetic fields $\gamma=5$ and $\gamma=11$.

Contour plots in Figures 8(a) and 8(b) for $\gamma=5$ show that the charge distribution in the ground state $(k=1)$ has the clearly pronounced even parity $\Sigma_{g}$, while the first excited state $(k=2)$ has the odd parity $\Sigma_{u}$. Parities of the ground and excited states are inverted as the magnetic field increases becoming equal to $\gamma=11$ and, therefore, contour plots for two lower states for $\gamma=11$ in Figure 8 are mutually interchanged. At the same time, after inversion, both these distributions are no longer pure; they exhibit weak mixing of two functions with different parities. A sharp transformation of charge distribution in two lowest energy states at the point $\gamma=10$ of Figure 8 (b) is similar to one, typical for the second order phase transition.

3.3. Off-Center Donor in Elongated $Q R$. If the off-center donor is placed over the horizontal axis of an elongated $\mathrm{QR}$, the axial symmetry of the system continues to be conserved and therefore the projection of the angular momentum is still a "good" quantum number for the classification of the energy levels, unlike the parity of the wave function, which no longer can be used for this purpose. In Figures 9 and 10 we present results of calculations of the energies and the charge distributions for the donor placed at the point with polar coordinates $\rho_{i}=20 \mathrm{~nm}, \vartheta_{i}=0^{\circ}$ corresponding to the location at the rim of the crater. Comparing the energy dependencies 


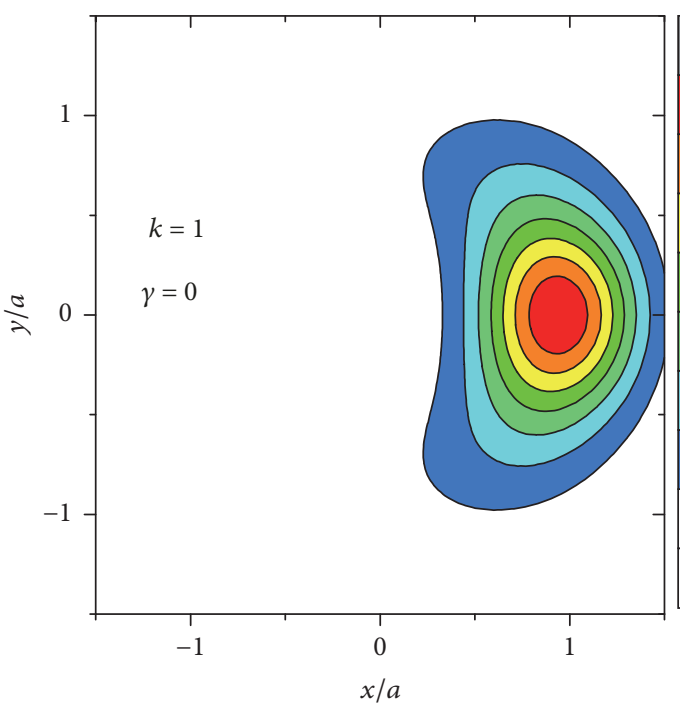

(a)

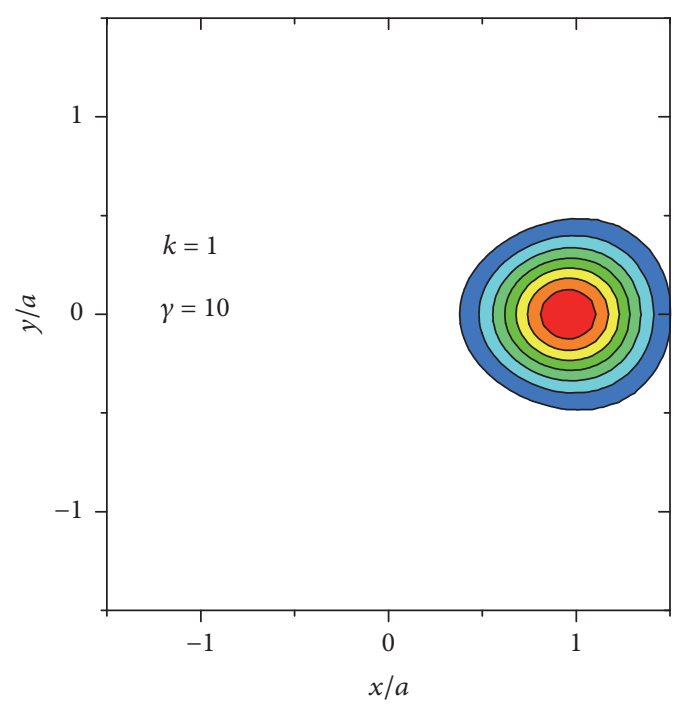

(c)
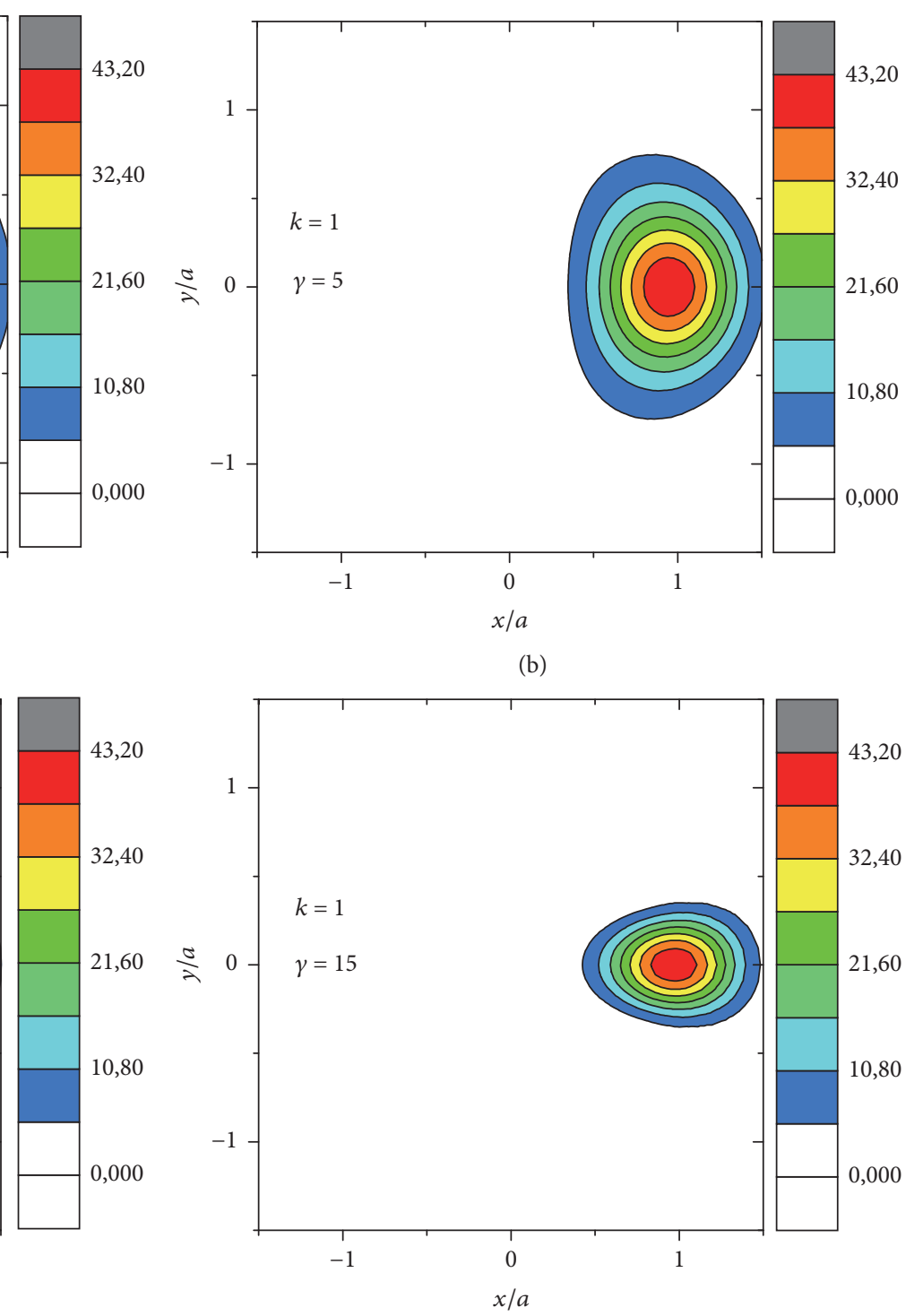

(d)

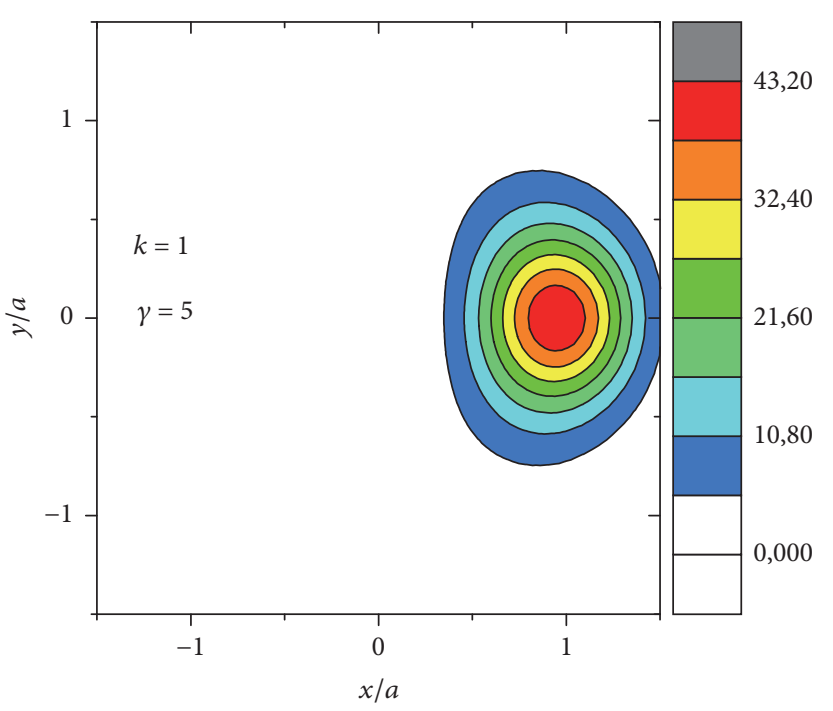

(b)

Figure 6: Charge distribution for off-center donor $\left(\rho_{i}=20 \mathrm{~nm}\right)$ in the ground state (a, b) and in the first excited state $(c, d)$ for $\gamma=0,5,10,15$ in circular QR.

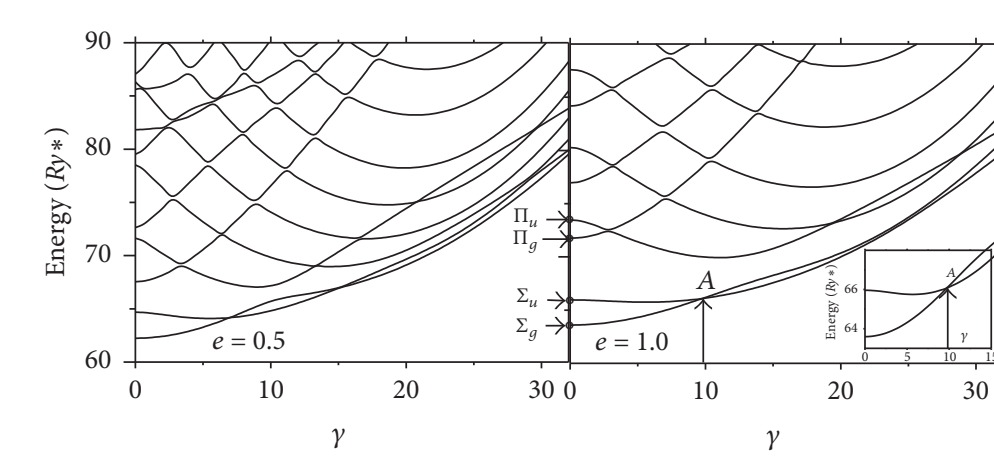

(a) (b)

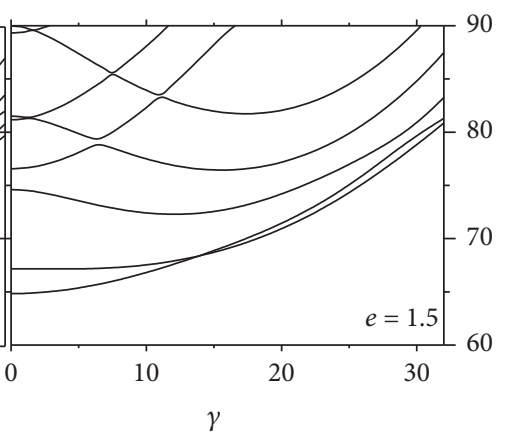

(c)

FIGURE 7: Energies of the on-center donor in elongated QR with parameters $a=20 \mathrm{~nm}, d_{r}=4 \mathrm{~nm}, d_{h}=2 \mathrm{~nm}$, and $e=0.5,1.0$, and 1.5, as functions of the external magnetic field. Inset in Figure 8(b) shows details of the first crossover of the lowest levels. 


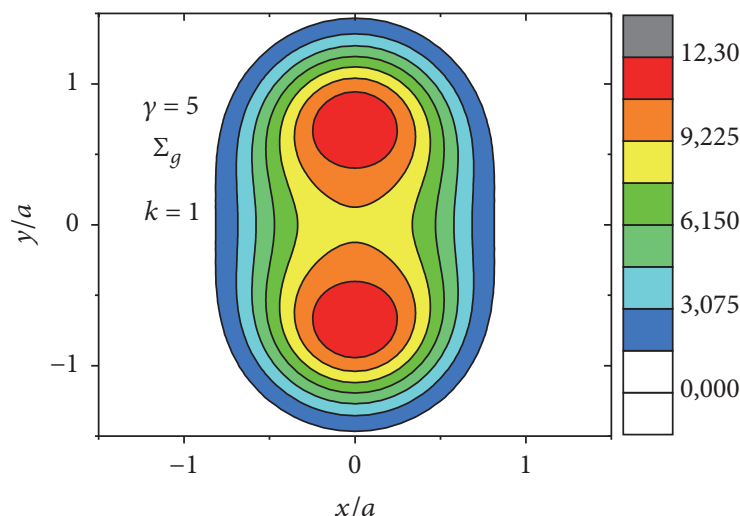

(a)

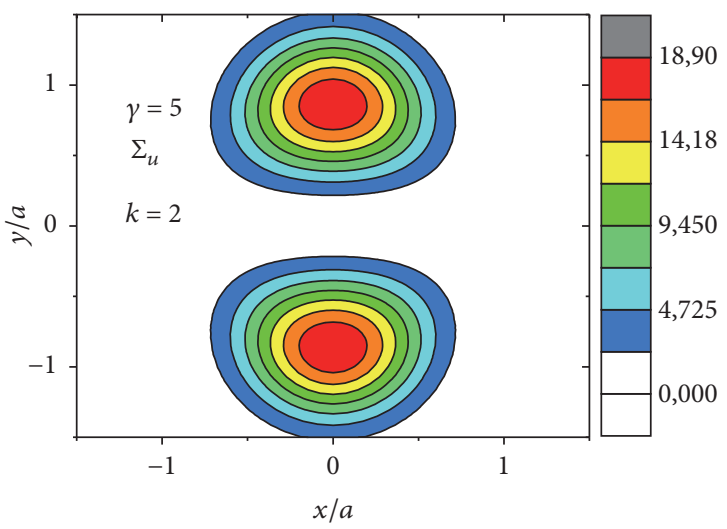

(b)

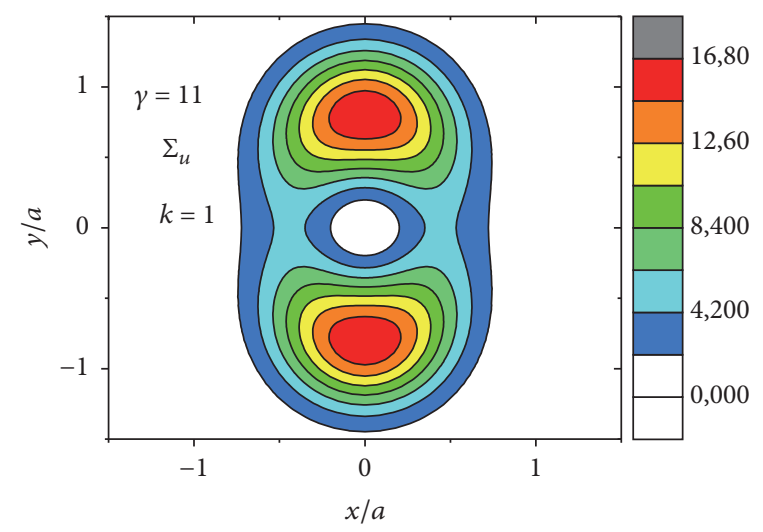

(c)

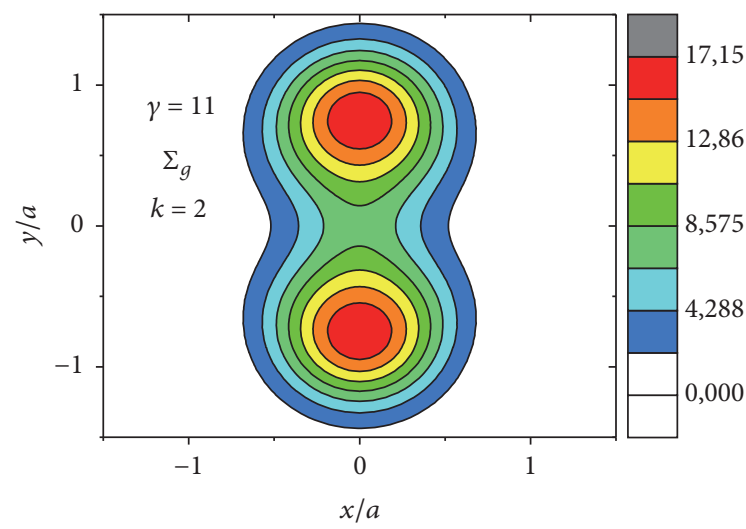

(d)

FIGURE 8: Charge distribution for on-center donor in the ground $(k=1)$ and in the first excited $(k=2)$ states for the magnetic field strengths $\gamma=5$ and $\gamma=11$ in elongated QR with the eccentricity parameter $e=1$.

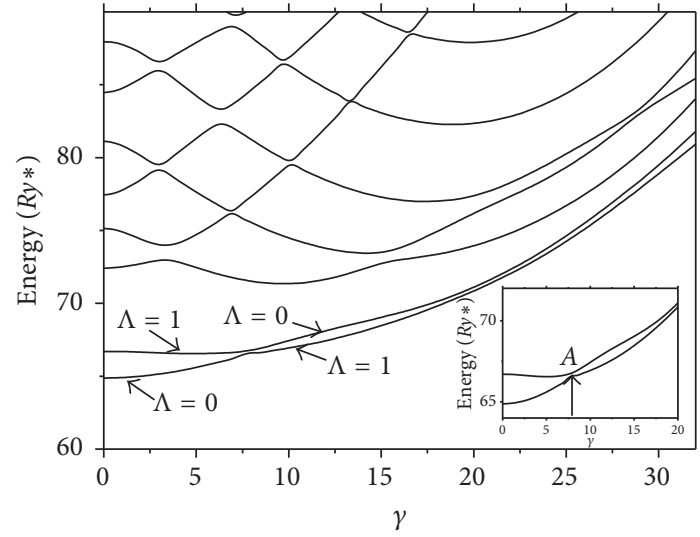

FIGURE 9: Energies of the off-axis donor in elongated QR with parameters $a=20 \mathrm{~nm}, d_{r}=4 \mathrm{~nm}, d_{h}=2 \mathrm{~nm}$, and $e=1.0$ as functions of the external magnetic field.

on the magnetic field for on-axis donor in Figure 7(b) with those for off-axis donor presented in Figure 9, one can notice general similitude between two sets of curves.

There are only small differences in the values of the energies and the positions of the crossovers of the curves.
Particularly, the energies in Figure 9 are higher than those in Figure 7 (b) by about $2 R y *$, the value that is consistent with the decreasing of the energy of the electron-donor attraction due to the increase of the averaged electron-donor separation related to the corresponding displacement of the donor position. Also, the first crossover of the energy dependencies of two lowest levels with $\Lambda=0$ and $\Lambda=1$ takes place in Figure 9 for the magnetic field that is slightly lower than one for the circular QR. Nevertheless, the variations of the charge distribution with the increase of the magnetic field in circular and elongated QRs are essentially different, unlike similar alterations of the energies. One can observe it comparing contour plots in Figure 8 for the circular QR with those presented in Figure 10 for elongated QR.

Contour plots in Figures 10(a) and 10(b) for two lowest states for $\gamma=0$ are similar to those in Figures 8(a) and 8(b), demonstrating a weak influence of the electron-donor donor electrostatic interaction. In fact, in the considered configuration the donor is located at the point with polar coordinates $\rho_{i}=20 \mathrm{~nm}, \varphi_{i}=0$, while the electron in these states is mainly sited close to the points $\rho=10 \mathrm{~nm}$, $\varphi= \pm 90^{\circ}$ and the averaged separation between them is sufficiently large. As the magnetic field is increased, the induced diamagnetic confinement diminishes this separation 


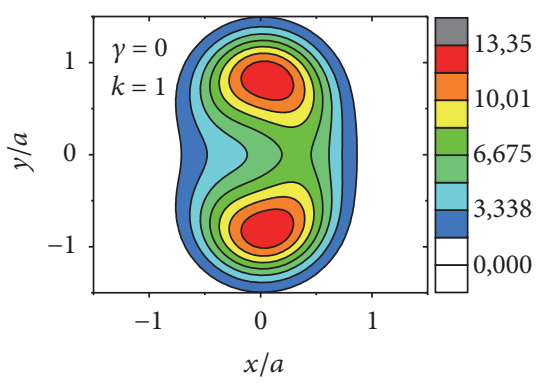

(a)

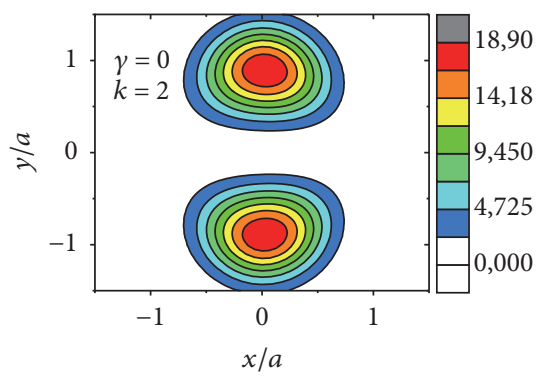

(d)

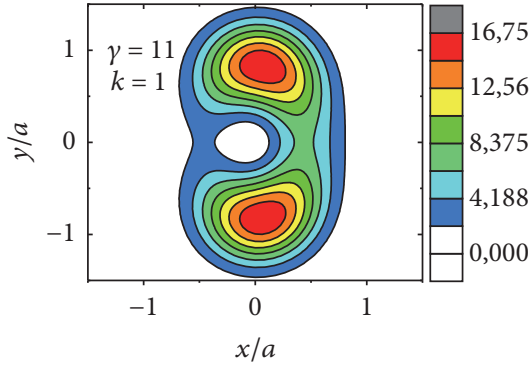

(b)

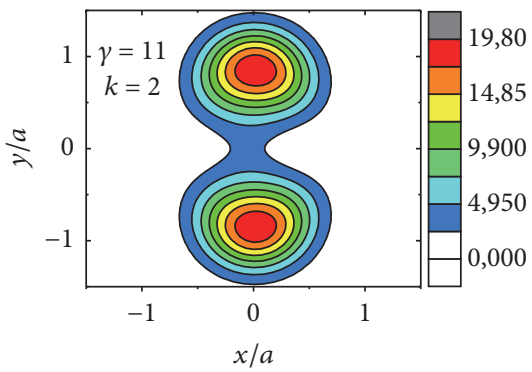

(e)

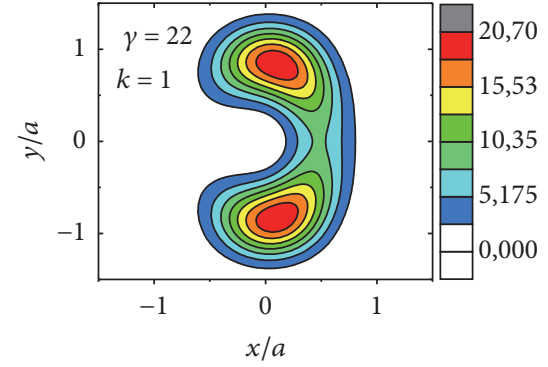

(c)

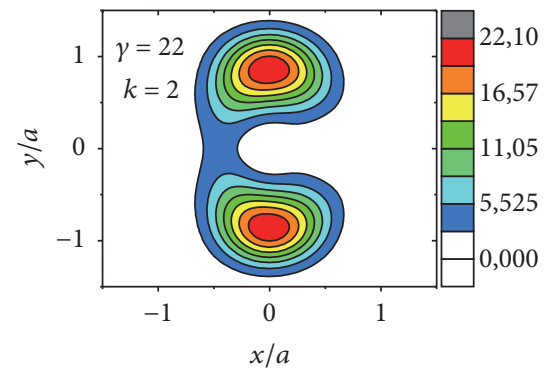

(f)

FIGURE 10: Charge distribution in elongated QR with the eccentricity parameter $e=1$ induced by off-axis donor $\left(\rho_{i}=20 \mathrm{~nm}\right)$ in the ground and in the first excited states for the magnetic fields $\gamma=0, \gamma=11$, and $\gamma=22$.

and provides a modification of the charge distribution mixing partially the wave functions of two lowest states. However, the magnetic field does not show any drastic alteration in the charge distribution because of absence of the high symmetry in this configuration, unlike the case of on-axis donor.

3.4. Effect of the Electric Field on Off-Axis Donor in Circular $Q D$. The excessive electron, released from the donor, located in volcano-shaped QD, is mainly located within a narrow region along its rim, constrained by the structural confinement. Energy gaps between states with different angular momenta in such arrangement are relatively small and therefore they become mixed in the presence of the external magnetic and electric fields. The electron in the ground state is located mainly at the region of the rim at shortest distance from the donor and the binding energy of this state is about $2 R y * \sim 4 \mathrm{meV}$. One can estimate that the electric field required for gaining this energy and for transferring the electron toward the opposite side of the rim is about $10 \mathrm{kV} / \mathrm{cm}$, and the charge distribution in the QD is induced by a donor located at the rim.

Such field could be able to modify drastically the charge distribution inside the QD, originating a giant dipole momentum. In Figures 11(a) and 11(b) we display the energies of some lower levels as functions of the electric field for the zero -magnetic field case $(\gamma=0)$ and in the presence of a strong magnetic field $\gamma=20(B \simeq 16 T)$. The slopes of these curves define the dipole momentum of the corresponding configuration. Critical electric fields, for which the slopes of curves in Figure 11 are changed abruptly, provide a drastic change of the charge distribution inside the QR and the polarizability of the structure. The adiabatic potential of the excessive electron resulting from the structural confinement, electron-donor interaction, and the external electric field has, along with the crater's rim, two minima: one at the region close to the donor and the other on the opposite side of the crater, originated by the electric field. When the increasing electric field attains the critical value, the second minimum becomes deeper than the first one. It is seen that the critical value of the electric field in the case of $\gamma=0$ is smaller than for $\gamma=20$ and the change of the slope in the first case is essentially smoother than in the presence of the strong magnetic field.

In Figures 12(a)-12(d) we present the contour plots of the charge distributions in a QR induced by the off-axis donor for three different values of the external electric field, in the presence of the magnetic field $\gamma=0$ (Figures 12(c)-12(e)) and $\gamma=20$ (Figures 12(f)-12(h)). One can observe that, as in the zero-magnetic-field case $(\gamma=0)$, the maximum of the density of the charge distribution shifts substantially to the left as the electric field increases. On the contrary, for $\gamma=20$ the maximum jumps abruptly as the electric field becomes superior to $3.5 \mathrm{kV} / \mathrm{cm}$. This result shows that the magnetic field obstructs the electric polarizability and it can be used to control the electric properties of the structure.

3.5. Effect of the Electric Field on Off-Axis Donor in Elongated $Q D$. One can expect similar effects for elongated QRs, but less pronounced. Really, elongated QRs have no longer central symmetry and therefore the mobility of the electron along the crater's rim under applied external electric field is more constrained. In this case it is necessary to apply a stronger electric field in order to achieve an essential redistribution of the probability density. In Figure 12 we show the results of calculation for elongated QR with the geometrical parameters 


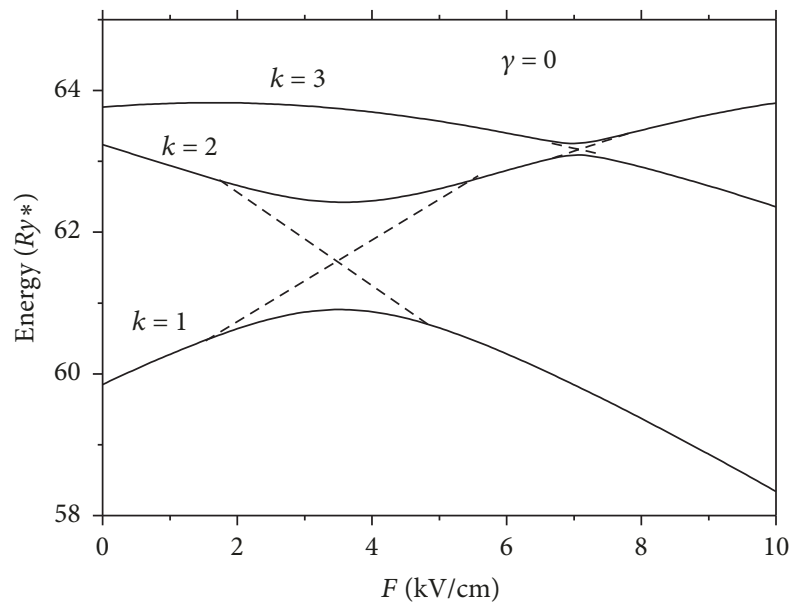

(a)

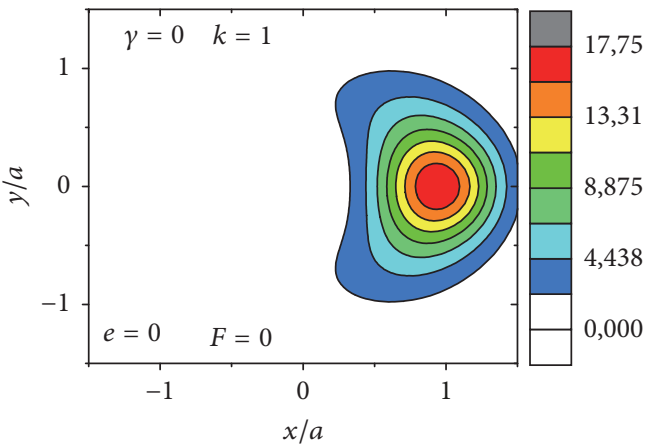

(c)

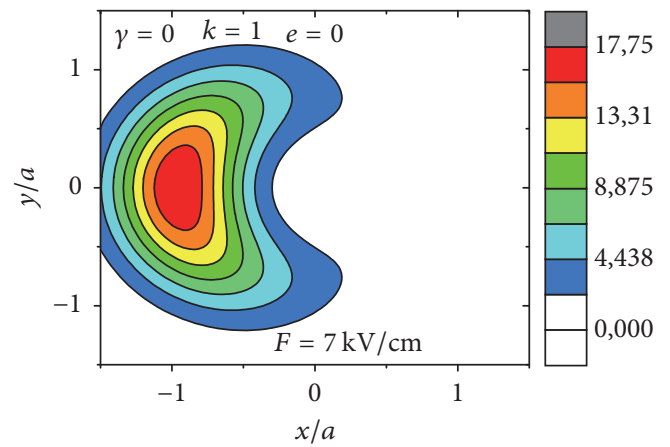

(e)

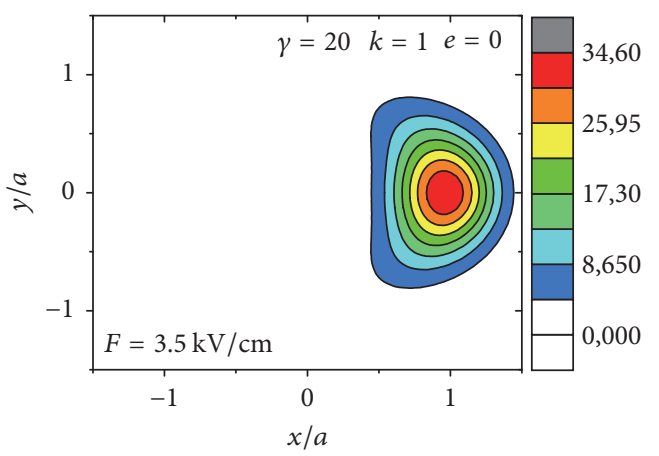

(g)

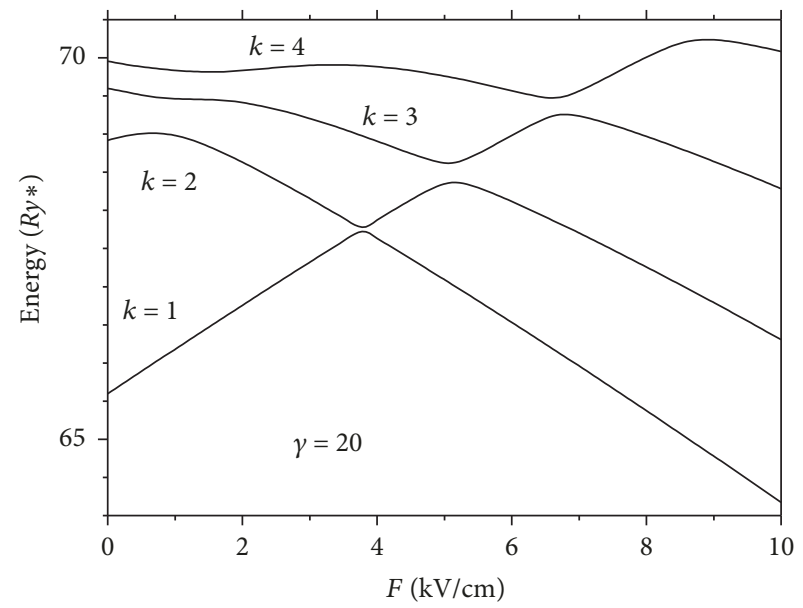

(b)

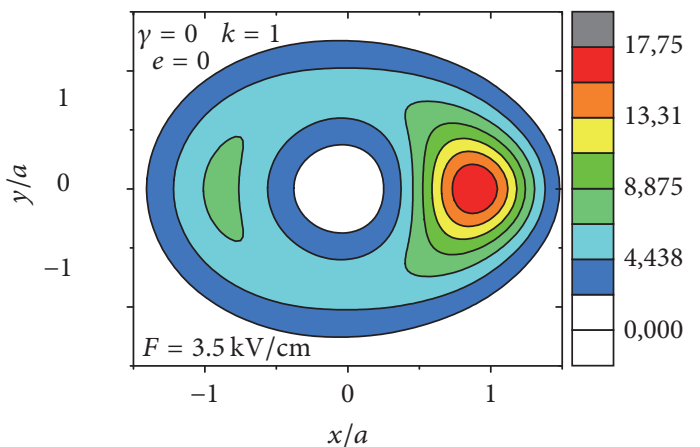

(d)

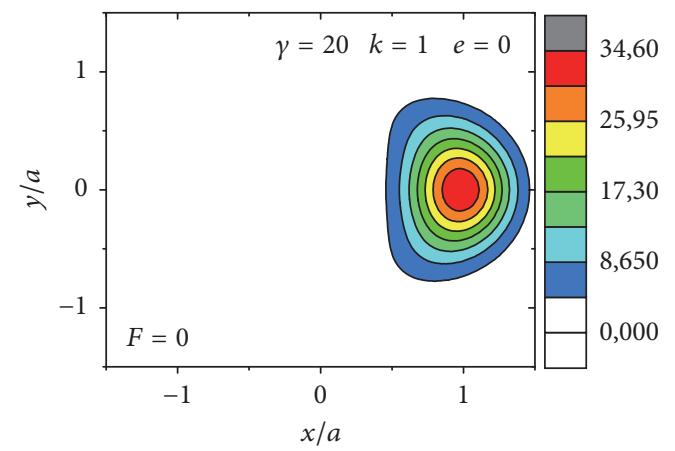

(f)

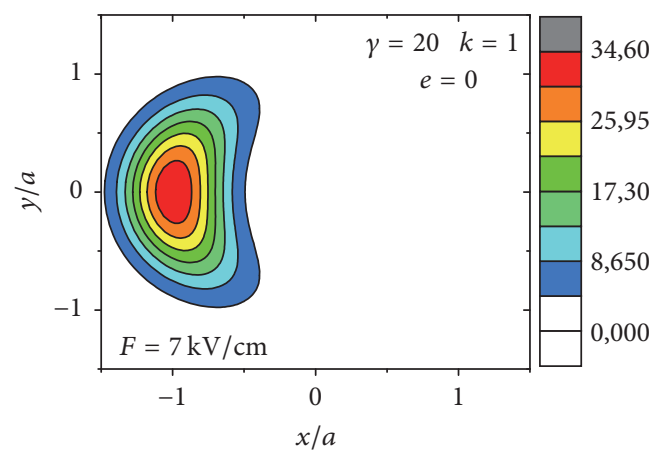

(h)

FIGURE 11: Lower energies of donor located at the crater's rim of the circular QR as functions of the electric field in the cases (a) zero-magnetic field and (b) $\gamma=20$ and contour plots for corresponding charge distributions. 


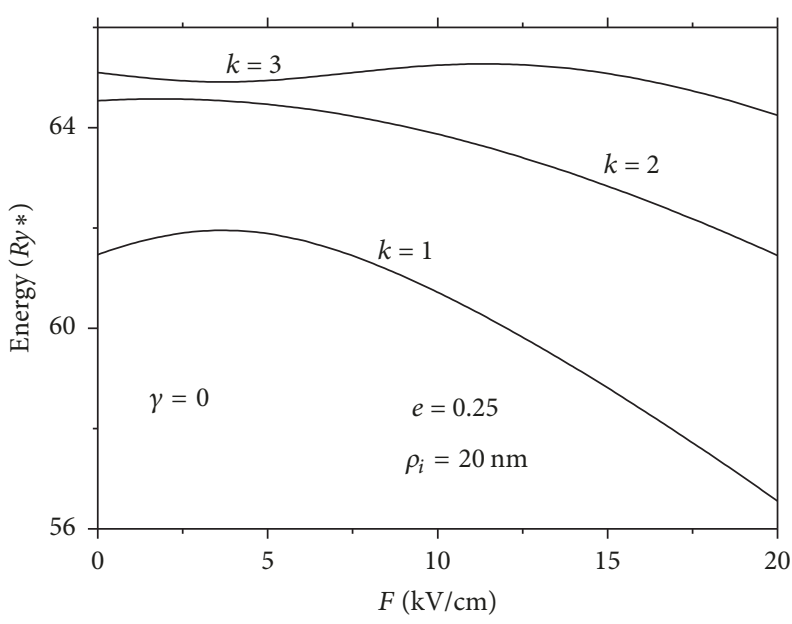

(a)

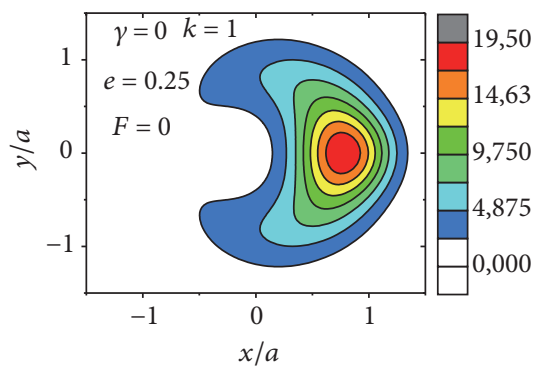

(c)

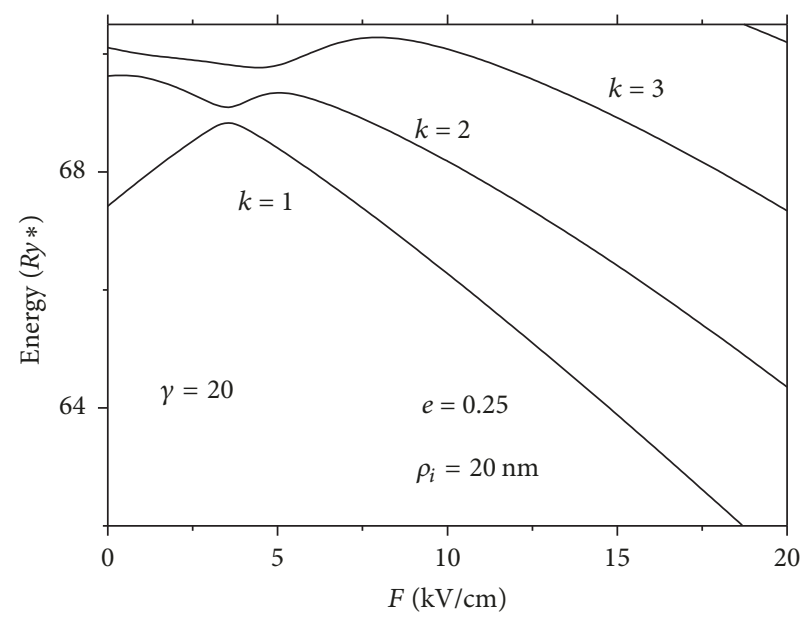

(b)

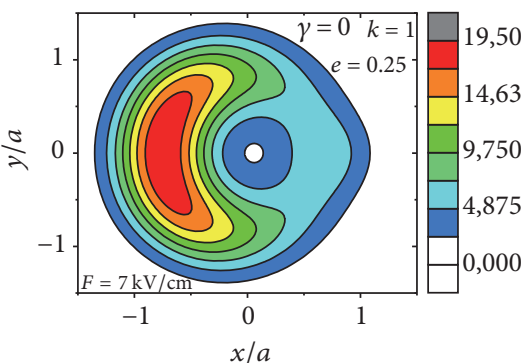

(d)

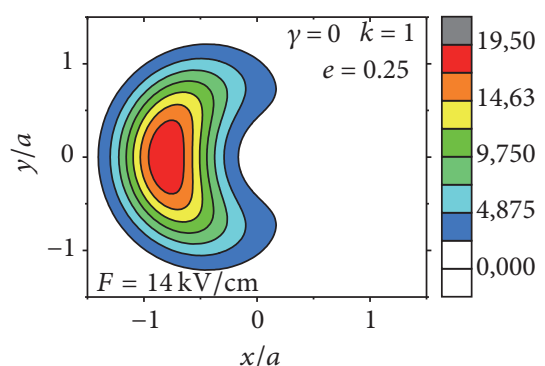

(e)

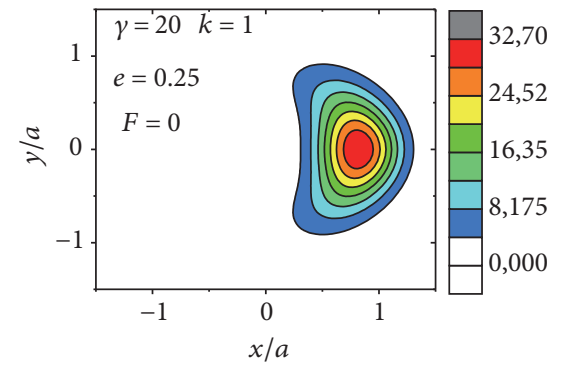

(f)

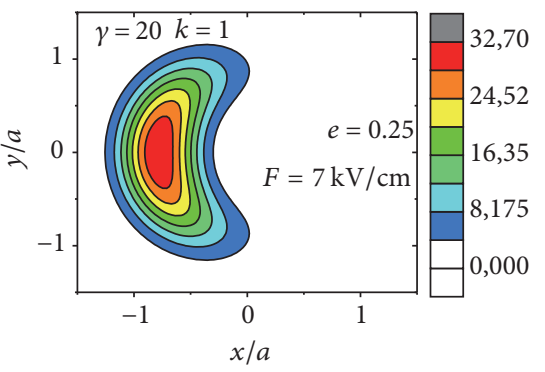

(g)

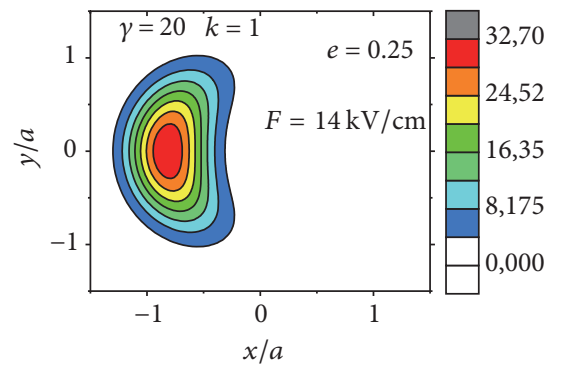

(h)

FIGURE 12: Lower energies of donor located at the crater's rim of the elongated QR as functions of the electric field in the cases (a) zeromagnetic field and (b) $\gamma=20$ and (c-f) contour plots for charge distributions.

$a=20 \mathrm{~nm}$ and $e=0.25$ similar to those presented in Figure 11 for a circular $\mathrm{QR}$.

One can observe a general similarity of Figures 11 and 12 with one essential difference in the scale of the electric field.
It is clear that the electric field required for an alteration of the electron orbits configurations in elongated QD is larger due to lack of the symmetry in comparison with the one in circular structure. 


\section{Conclusions}

In summary, we have proposed a simple theoretical method for analyzing the energy spectrum and the charge distribution induced by a shallow donor in an InAs/GaAs volcano-shaped quantum ring in the presence of the mutually orthogonal electric and magnetic fields taking parabolicity of the conduction band in the framework of the Kane model. The method allows us to find explicitly two-dimensional adiabatic confinement potential associated with a particular profile of the InAs layer. In this work, for simulating the profile of the elongated volcano-shaped quantum dots, we select a function with four parameters, which define the rim, the central hole, rim radii and height dimensions, and the eccentricity. For the solution of the resulting two-dimensional Schrödinger equation for the electron released from the donor, we use the double Fourier-Bessel series expansion. Comparing donor energies obtained in the effective mass approximation and for the Kane model, we find that the donor energies in the last case are noticeably lower in comparison with those obtained earlier in the effective mass approximation.

We present analysis of the influence of the external magnetic field on the energy spectra of both on- and off-axis in circular and elongated volcano-shaped quantum dots. The results of energies dependencies on the magnetic field for onaxis donor in a circular structure exhibit general similitude with those obtained previously in the effective mass approximation for quasi-one-dimensional models with periodical Aharonov-Bohm oscillations and multiple crossovers. A displacement of the donor from the axis, as well transformations of the morphology of the ring, produces quenching of the oscillations of the lower levels and the disappearance of the gaps between them. In addition, we show that any change in the system related to the lack of the symmetry originates from more significant alteration of the charge distribution inside the quantum dot than those in the energy spectrum.

Also, we analyze the effect of the electric field on the energy spectrum and the charge distribution induced by the off-axis donor. We found that when the increasing electric field exceeds a critical value, the charge distribution inside the structure induced by the excessive electron changes sharply generating giant dipole momentum. We show, in addition, that this process can be controlled by the external magnetic field applied along the crystal growth direction.

\section{Data Availability}

The data used to support the findings of this study are available from the corresponding author upon request.

\section{Conflicts of Interest}

The authors declare that they have no conflicts of interest.

\section{Acknowledgments}

This work was financed by the "Universidad Industrial de Santander (UIS)" through the Vicerrectoría de Investigación y Extensión (VIE), DIEF de Ciencias (Cod. 5124), and the "Patrimonio Autónomo del Fondo Nacional de Financiamiento para la Ciencia, la Tecnología y la Innovación Francisco Jose De Caldas-Contrato RC no. 275-2011” and the Cod. no. 1102-05-16923 subscribed with "Colciencias."

\section{References}

[1] A. Lorke and R. J. Luyken, "Many-particle ground states and excitations in nanometer-size quantum structures," Physica B: Condensed Matter, vol. 256-258, pp. 424-430, 1998.

[2] A. Lorke, R. J. Luyken, A. O. Govorov, J. P. Kotthaus, J. M. Garcia, and P. M. Petroff, "Spectroscopy of Nanoscopic Semiconductor Rings," Physical Review Letters, vol. 84, no. 10, pp. 2223-2226, 2000.

[3] A. Emperador, M. Pi, M. Barranco, and A. Lorke, "Far-infrared spectroscopy of nanoscopic InAs rings," Physical Review B: Condensed Matter and Materials Physics, vol. 62, no. 7, pp. 4573$4577,2000$.

[4] R. J. Warburton, C. Schulhauser, D. Haft et al., "Giant permanent dipole moments of excitons in semiconductor nanostructures," Physical Review B: Condensed Matter and Materials Physics, vol. 65, no. 11, 2002.

[5] B. Alén, J. Martínez-Pastor, D. Granados, and J. M. García, "Continuum and discrete excitation spectrum of single quantum rings," Physical Review B: Condensed Matter and Materials Physics, vol. 72, no. 15, 2005.

[6] T. Kuroda, T. Mano, T. Ochiai et al., "Optical transitions in quantum ring complexes," Physical Review B: Condensed Matter and Materials Physics, vol. 72, no. 20, 2005.

[7] G. Bastard, "Hydrogenic impurity states in a quantum well: A simple model," Physical Review B: Condensed Matter and Materials Physics, vol. 24, no. 8, pp. 4714-4722, 1981.

[8] R. L. Greene and K. K. Bajaj, "Energy levels of hydrogenic impurity states in GaAs-Gal-xAlxAs quantum well structures," Solid State Communications, vol. 45, no. 9, pp. 825-829, 1983.

[9] R. L. Greene and K. K. Bajaj, "Effect of magnetic field on the energy levels of a hydrogenic impurity center in GaAs," Physical Review B: Condensed Matter and Materials Physics, vol. 31, no. 2, pp. 913-918, 1985.

[10] S. Fraizzoli, F. Bassani, and R. Buczko, "Shallow donor impurities in GaAs-Gal-xAlxAs quantum-well structures: role of the dielectric-constant mismatch," Physical Review B: Condensed Matter and Materials Physics, vol. 41, no. 8, pp. 5096-5103, 1990.

[11] J. M. Shi, F. M. Peeters, and J. T. Devreese, "Shallow donor impurities in GaAs/AlxGal-xAs superlattices in a magnetic field," Physical Review B: Condensed Matter and Materials Physics, vol. 50, no. 20, pp. 15182-15190, 1994.

[12] S. V. Branis, G. Li, and K. K. Bajaj, "Hydrogenic impurities in quantum wires in the presence of a magnetic field," Physical Review B: Condensed Matter and Materials Physics, vol. 47, no. 3, pp. 1316-1323, 1993.

[13] A. Montes, C. A. Duque, and N. Porras-Montenegro, "Density of shallow-donor impurity states in rectangular cross section GaAs quantum-well wires under applied electric field," Journal of Physics: Condensed Matter, vol. 10, no. 24, pp. 5351-5358, 1998.

[14] J. Zhu, "Exact solutions for hydrogenic donor states in a spherically rectangular quantum well," Physical Review B: Condensed Matter and Materials Physics, vol. 39, no. 12, pp. 8780-8783, 1989.

[15] J. Zhu, J. Xiong, and B. Gu, "Confined electron and hydrogenic donor states in a spherical quantum dot of GaAs- $\mathrm{Ga}_{1-x} \mathrm{Al}_{x} \mathrm{As}$," Physical Review B: Condensed Matter and Materials Physics, vol. 41, no. 9, pp. 6001-6007, 1990. 
[16] F. J. Ribeiro and A. Latgé, "Impurities in a quantum dot: A comparative study," Physical Review B: Condensed Matter and Materials Physics, vol. 50, no. 7, pp. 4913-4916, 1994.

[17] B. Szafran, J. Adamowski, and S. Bednarek, "Electron-electron correlation in quantum dots," Physica E: Low-dimensional Systems and Nanostructures, vol. 5, no. 3, pp. 185-195, 2000.

[18] V. M. Fomin, V. N. Gladilin, S. N. Klimin, J. T. Devreese, N. A. Kleemans, and P. M. Koenraad, "Theory of electron energy spectrum and Aharonov-Bohm effect in self-assembled "Physical Review B: Condensed Matter and Materials Physics, vol. 76, no. 23, 2007.

[19] F. M. Peeters and V. A. Schweigert, "Two-electron quantum disks," Physical Review B: Condensed Matter and Materials Physics, vol. 53, no. 3, pp. 1468-1474, 1996.

[20] M. Korkusiński and P. Hawrylak, "Electronic structure of vertically stacked self-assembled quantum disks," Physical Review B: Condensed Matter and Materials Physics, vol. 63, no. 19, 2001.

[21] D. Mikhailov, J. H. Marín, and F. García, "Off-axis donors in quasi-two-dimensional quantum dots with cylindrical symmetry," Physica Status Solidi (b), vol. 242, no. 8, pp. 1636-1649, 2005.

[22] K. L. Janssens, F. M. Peeters, and V. A. Schweigert, "Magneticfield dependence of the exciton energy in a quantum disk," Physical Review B: Condensed Matter and Materials Physics, vol. 63, no. 20, 2001.

[23] E. Kane, "Band structure of indium antimonide," Journal of Physics and Chemistry of Solids, vol. 1, no. 4, pp. 249-261, 1957.

[24] Y. M. Li, H.-M. Lu, O. Voskoboynikov, C. P. Lee, and S. M. Sze, "Dependence of energy gap on magnetic field in semiconductor nano-scale quantum rings," Surface Science, vol. 532-535, pp. 811-815, 2003.

[25] I. Filikhin, V. M. Suslov, and B. Vlahovic, "Electron spectral properties of the InAs/GaAs quantum ring," Physica E: Lowdimensional Systems and Nanostructures, vol. 33, no. 2, pp. 349354, 2006.

[26] L. F. García, S. Y. Revinova, and I. D. Mikhailov, "Band nonparabolicity effect on spectral properties of quantum ring," Superlattices and Microstructures, vol. 103, pp. 151-160, 2017.

[27] S. Bietti, S. Sanguinetti, C. Somaschini et al., "Fabrication of GaAs quantum dots by droplet epitaxy on $\mathrm{Si} / \mathrm{Ge}$ virtual substrate," IOP Conference Series: Materials Science and Engineering, vol. 6, no. 1, Article ID 012009, 2009.

[28] T.-C. Lin, C.-H. Lin, H.-S. Ling et al., "Impacts of structural asymmetry on the magnetic response of excitons and biexcitons in single self-assembled $\operatorname{In}(\mathrm{Ga})$ As quantum rings," Physical Review B: Condensed Matter and Materials Physics, vol. 80, no. 8, Article ID 081304, 2009.

[29] P. Boonpeng, W. Jevasuwan, S. Suraprapapich, S. Ratanathammaphan, and S. Panyakeow, "Quadra-quantum dots grown on quantum rings having square-shaped holes: Basic nanostructure for quantum dot cellular automata application," Microelectronic Engineering, vol. 86, no. 4-6, pp. 853-856, 2009.

[30] G. Linares-García, L. Meza-Montes, E. Stinaff et al., "Optical properties of a quantum dot-ring system grown using droplet epitaxy," Nanoscale Research Letters, vol. 11, no. 1, article no. 309, 2016.

[31] H. D. Kim, R. Okuyama, K. Kyhm et al., "Observation of a Biexciton Wigner Molecule by Fractional Optical AharonovBohm Oscillations in a Single Quantum Ring," Nano Letters, vol. 16, no. 1, pp. 27-33, 2016. 

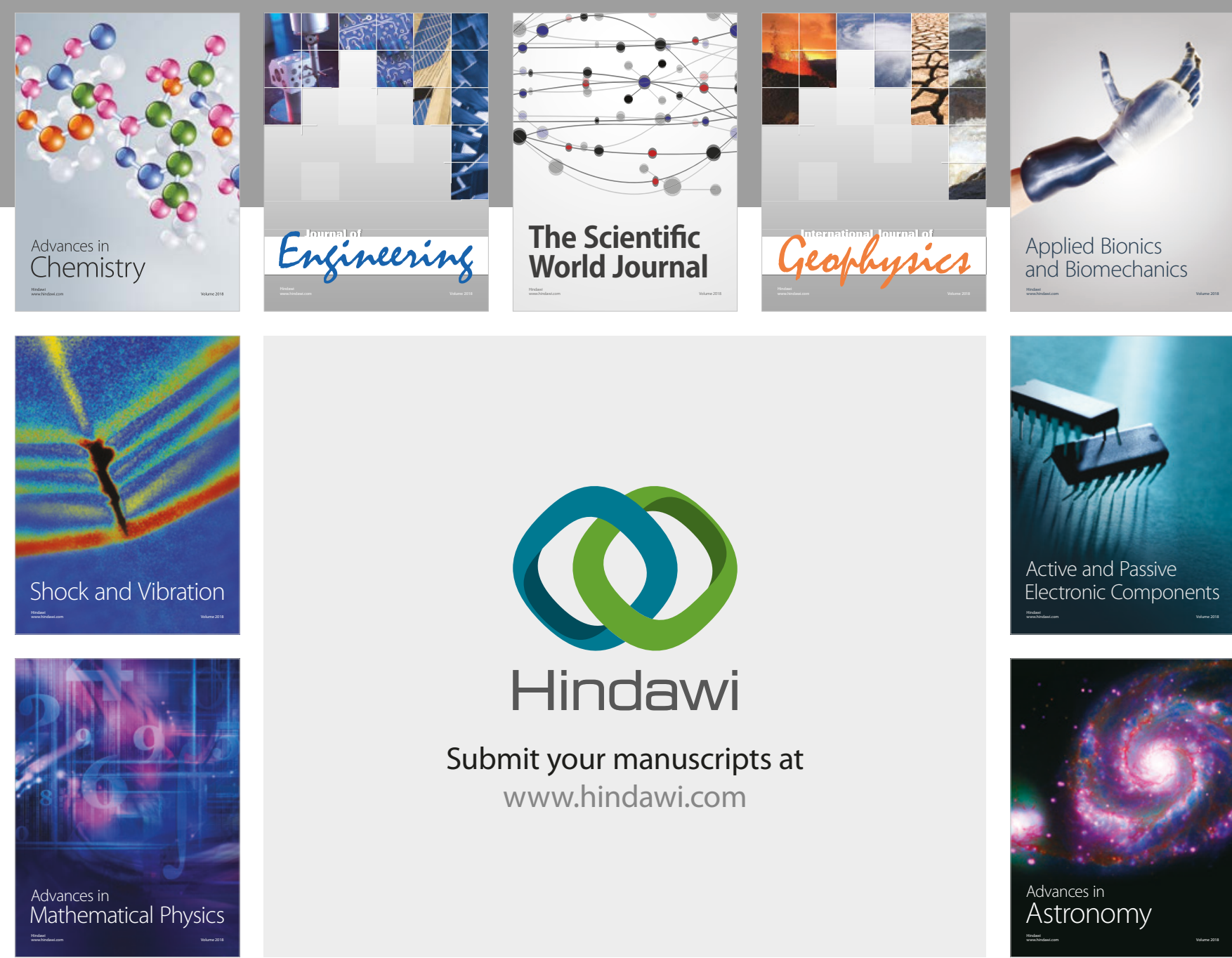

Submit your manuscripts at

www.hindawi.com

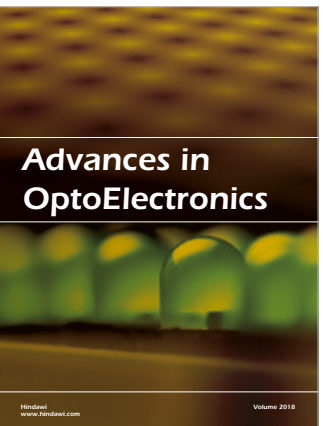

\section{Rotcting Machinery}
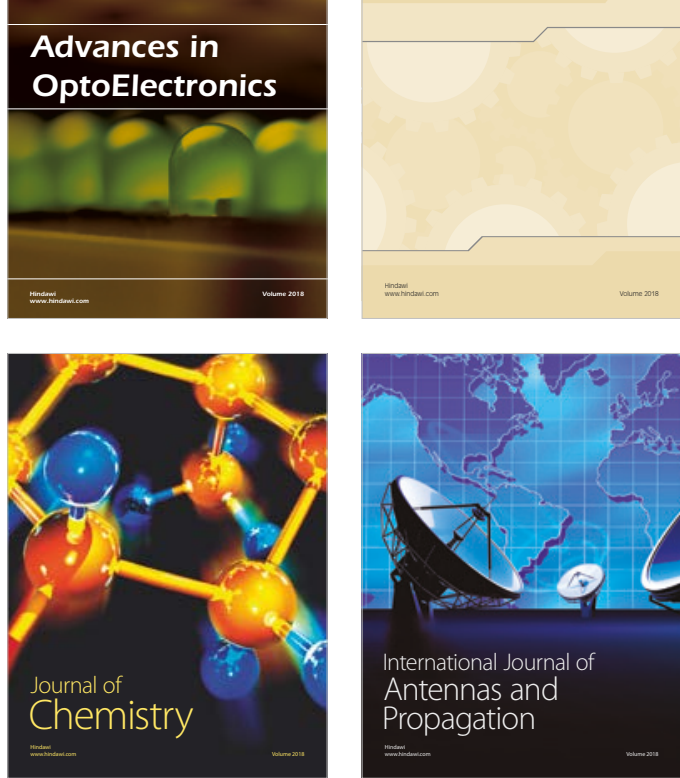

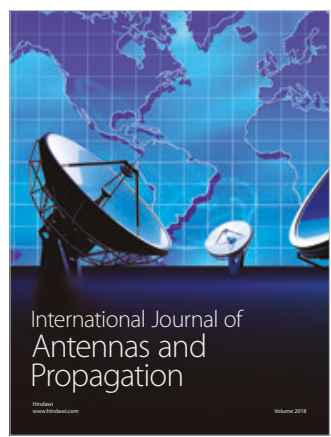

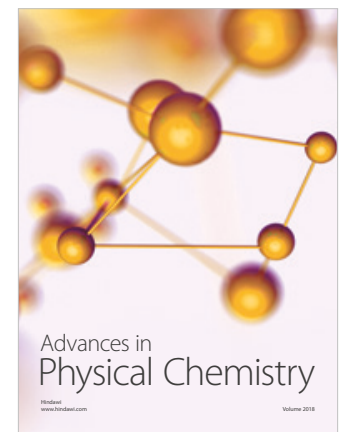

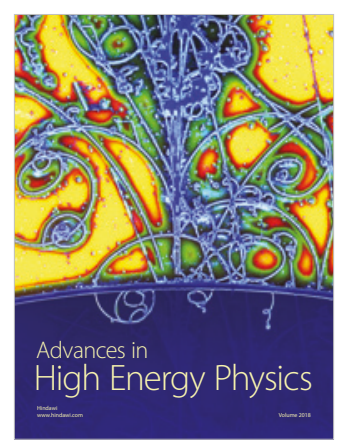

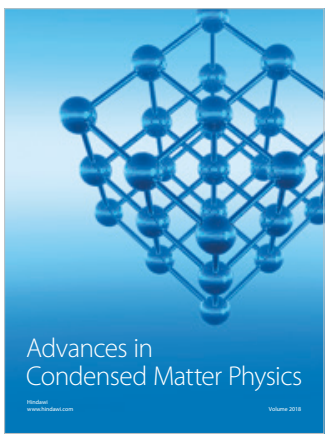

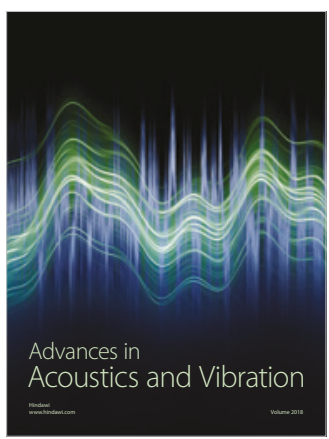

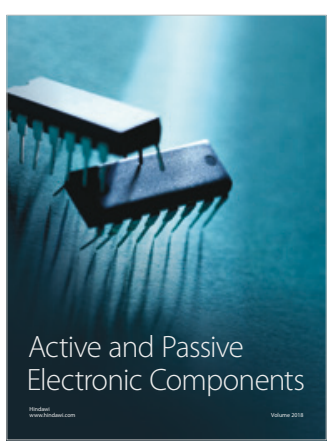
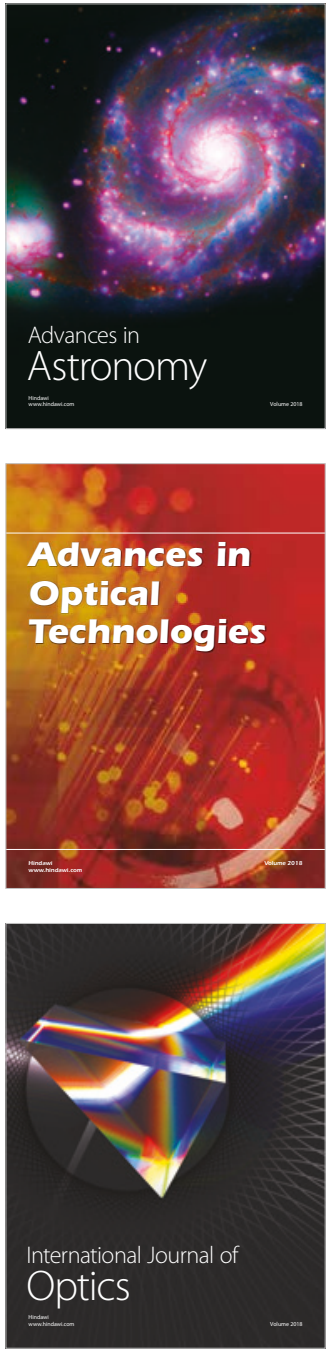